\section{Military Technical College Kobry El-Kobbah, Cairo, Egypt}

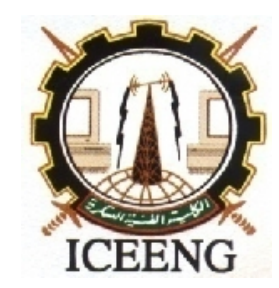

\section{$7^{\text {th }}$ International Conference on Electrical Engineering ICEENG 2010}

\title{
Ballistic Navigation Solution Using GPS/INS Integration
}

\author{
By
}

I. I. Arfaa ${ }^{* *}, \quad$ Y. Z. Elhalwagy ${ }^{* *}, \quad$ A. M. Abdelmaged ${ }^{* *}$, and R. A. Elbordany ${ }^{* *}$

\section{$\underline{\text { Abstract: }}$}

This paper demonstrates the importance of the INS/GPS/GLONASS integration into ballistic navigation solution. The SINS uses (ENU) frame and the integrated system uses position and velocity as measurements. The system model of the integrated system for Kalman filtering are derived and modeled as 27-states. This state estimation system shown clearly the application of fundamental modeling and filtering techniques. The simulation is built on the integrated system INS/GPS/GLONASS and the trajectory generator data. From the results we found that parameters of estimated gyro errors such as vertical and east gyro drifts, and also estimated east accelerometer bias are not observables.

\section{Keywords:}

Aided Navigation System, Kalman Filter, SDINS

\footnotetext{
*** Egyptian Armed Forces

${ }^{* *}$ Egyptian Armed Forces

${ }^{* *}$ Egyptian Armed Forces

${ }^{* *}$ Egyptian Armed Forces
} 


\section{Introduction:}

Inertial navigation systems exhibit position errors that tend to increase with time in an unbounded manner. This degradation is due, in part; to errors in the initialization of the inertial measuring unit and inertial sensor imperfections such as accelerometer bias and gyroscope drift [1-13]. One way of mitigating this growth and bounding the errors is to update the inertial system periodically with external aided. Therefore, when the external aided is available on the ground or on board the navigating vehicle, the accuracy of the navigation process can be greatly improved through external position and velocity measurements. Therefore, in order to achieve mission accuracy requirements, further inertial navigation systems will most likely include more navigation sensors and subsystem modes.

Integrated INS/GPS is a promising technology for the High Speed Civil Transport (HSCT) and the return and landing of a manned space vehicle. The presence of failures in navigation sensors can cause the determination of an erroneous vehicle state estimate, which includes position, attitude, and their derivatives [10]. Missile flight control systems rely on sensor inputs to determine the vehicle state. In the case of integrated Inertial/NAVSTAR Global Positioning System (GPS), sensor failures could occur in the on-board inertial sensors or in the GPS measurements [9]. The synergistic use of both GPS and the INS allows for highly reliable fault detection and isolation of sensor failures [2]. In order to combine INS and GPS data by using filtering techniques, we have two approaches the 1st one is the standard Kalman filter, which processes the data from different systems in one step, is called the centralized Kalman filter. The 2nd one is the decentralized filtering technique, in which two Kalman filters are formulated [14]. One is the GPS filter, which is used as a local filter and processes GPS data only. The other one is the INS filter which is considered as the master filter and estimates position, velocity, and attitude along the trajectory. In this paper, the strap dawn INS uses (ENU) frame and the integrated system uses position and velocity as measurements is presented. The system model of the integrated system for Kalman filtering are derived and modeled as 27 -states. The simulation is built on the integrated system INS/GPS/GLONASS and the trajectory generator data. The paper terminates with conclusions.

\section{Mission Planning:}

The basic program objective is to fly a typical mission profile as shown in fig (1) with an on target accuracy of $\pm 200 \mathrm{~m}$. The flight path profile in the active phase approximately two minutes. The reminder of 15 minute flight is a ballistic trajectory. The commnd of separation will arrive after the 
thrust termination of the engine. The payload objects are deployed in reentry phase over a distance about $15 \mathrm{~km}$ from the ground. To meet target accuracy, the navigation system must provide accurate position and velocity input to the overall Guidance Navigation Control System (GNCS) for the active phase of flight [5]. At the end of active phase, the program has no other propellant capability so the GNCS has no more control over the delta velocity of the payload objects. The navigation solution must therefore be highly accurate for the first two minutes of flight (active phase) and thereafter, it provides useful information about the shape of the trajectory actually flown.

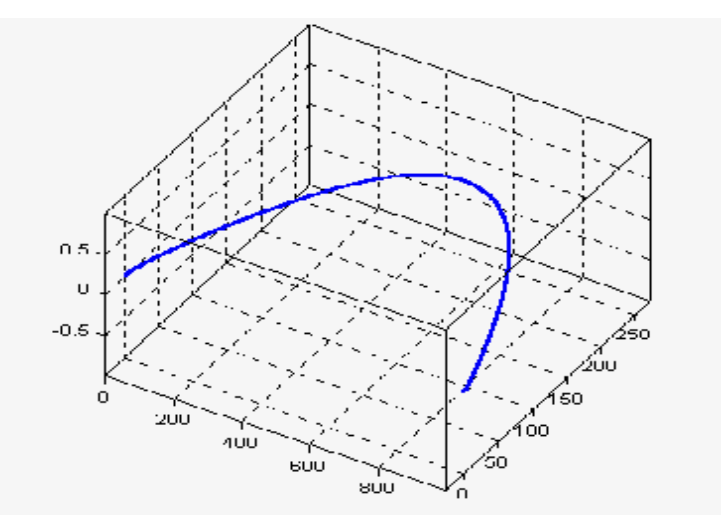

Figure (1): Mission Profile

\section{On-Board Computer Software}

The On-Board Software (OBS) is a complex system with many functions. It performs the following functions as shown in Fig (2): interface with ground support equipment, the majority of system test and checkout, ground processing, launch and all of the navigation, guidance, and control and sequencing functions required for flight [5,15]. The OBS development was comprised of 3 major components: 1) the operating system, IMU digital signal processing code and ground alignment software; 2) ground checkout code developed uniquely for the program system; 3) the flight guidance, navigation, control and sequencing code.

The flight navigation routines are dependent on inputs from the IMU sensors (gyros, accelerometers). The compensated sensor outputs, i.e. incremental angles and velocities, are accumulated and made available at $256 \mathrm{~Hz}$. 


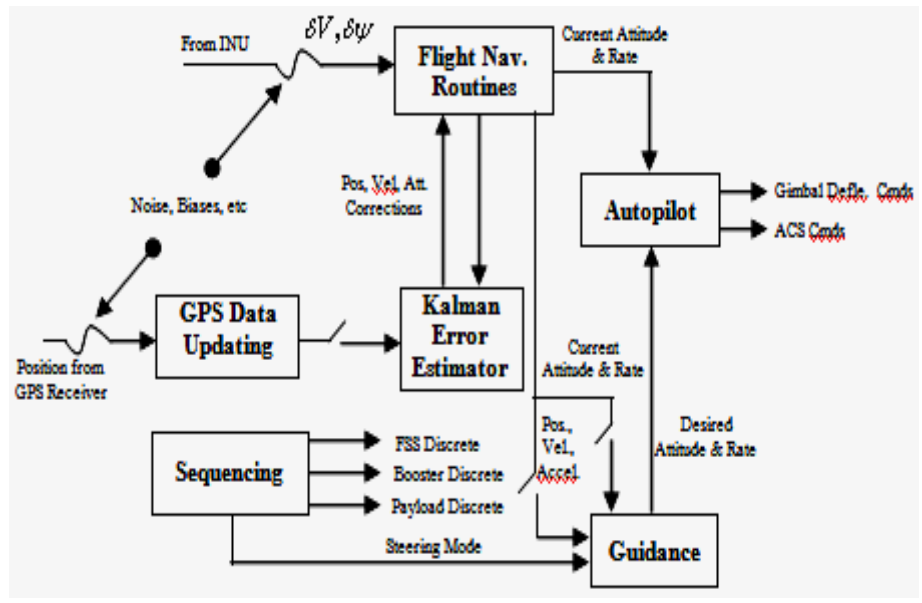

\section{Figure (2): OBS Flight Functional Block Diagram}

Additional compensations are also implemented in the flight inertial navigation algorithm to compensate for the estimated instrument parameters via the System Error Estimator (SEE) updates $[4,9]$. The SEE is a fifteen states Kalman filter algorithm that utilizes the position error, as 3 scalar measurements, between the propagated inertial position and GPS receiver solution. After the final implementation integrates the GPS information into the flight navigation solution via the Kalman filter: There are two mode of operation for the system: 1) ground test which assure the navigation system is healthy before launch and 2) navigation in flight. In the ground test we compute the system azimuth, level orientation and auxiliary sensor compensation through the ground alignment time. This creates the initial attitude reference and gyro and accelerometer biases and scale factors which are used at the time of (Go Inertial) for the flight navigation system [5]. At "Go Inertial", the flight navigation routines, the GPS Update Process and The Kalman filter are turned on and the system begins navigating.

\section{GPS/INS Integrated Model}

This section will demonstrate the integration of sensor and system dynamic data and their appropriate models using an optimal filter to create a robust, adaptable, easily reconfigurable state estimation system. A twenty seven state Kalman filter integration will be implemented in flight test mode to evaluate system performance. The filter integration approaches will be evaluated using data during flight test. The flight test data consisted of measurement of IMU, GPS, and trajectory generator data [16]. The general model and subsequent algorithm is developed in MATLAB for numerical testing. 
The results of this system are demonstrated through application to data from the trajectory mission and are presented in plots and simple animations.

\subsection{INS Error Model}

The geographic implementation of the inertial navigation position rate equations calculates latitude, longitude rate directly as a function of computed missile north and east velocity components in the navigation frame NEU and the position error can be modeled as follows:

$$
\begin{aligned}
& \delta \phi^{\&}=\frac{\delta V_{y}^{n}}{R_{M}+h} ; \\
& \delta k=\frac{\delta V_{x}^{n}}{\left(R_{N}+h\right) \cos \phi}+\frac{V_{x}^{n} \tan \phi \sec \phi}{R_{N}+h} \delta \phi \\
& \delta k^{\&}=\delta V_{z}^{n}
\end{aligned}
$$

The attitude error components can be written as [6]:

$$
\begin{aligned}
& \phi_{x}^{x}=-\frac{\delta V_{y}^{\mathrm{n}}}{\mathrm{R}_{\mathrm{M}}+\mathrm{h}}+\left(\Omega \sin \varphi+\frac{\mathrm{V}_{\mathrm{x}}^{\mathrm{n}} \tan \varphi}{\mathrm{R}_{\mathrm{N}}+\mathrm{h}}\right) \phi_{\mathrm{y}}^{\mathrm{n}}-\left(\Omega \cos \varphi+\frac{\mathrm{V}_{\mathrm{x}}^{\mathrm{n}}}{\mathrm{R}_{\mathrm{N}}+\mathrm{h}}\right) \phi_{z}^{\mathrm{n}}+\Delta \omega_{\mathrm{x}}^{\mathrm{n}} \\
& =-\Omega \sin \varphi \delta \varphi+\frac{\delta \mathrm{V}_{\mathrm{x}}^{\mathrm{n}}}{\mathrm{R}_{\mathrm{M}}+\mathrm{h}}-\left(\Omega \sin \varphi+\frac{\mathrm{V}_{\mathrm{x}}^{\mathrm{n}} \tan \varphi}{\mathrm{R}_{\mathrm{N}}+\mathrm{h}}\right) \phi_{\mathrm{x}}^{\mathrm{n}}-\frac{\mathrm{V}_{\mathrm{y}}^{\mathrm{n}}}{\mathrm{R}_{\mathrm{M}}+\mathrm{h}} \phi_{z}^{\mathrm{n}}+\Delta \omega_{\mathrm{y}}^{\mathrm{n}} \\
& (2)=\left(\Omega \cos \varphi+\frac{\mathrm{V}_{\mathrm{x}}^{\mathrm{n}} \sec ^{2} \varphi}{\mathrm{R}_{\mathrm{N}}+\mathrm{h}}\right) \delta \varphi+\frac{\tan \varphi}{\mathrm{R}_{\mathrm{N}}+\mathrm{h}} \delta \mathrm{V}_{\mathrm{x}}^{\mathrm{n}}+\left(\Omega \cos \varphi+\frac{\mathrm{V}_{\mathrm{x}}^{\mathrm{n}}}{\mathrm{R}_{\mathrm{N}}+\mathrm{h}}\right) \phi_{\mathrm{x}}^{\mathrm{n}}+\frac{\mathrm{V}_{\mathrm{y}}^{\mathrm{n}}}{\mathrm{R}_{\mathrm{M}}+\mathrm{h}} \phi_{\mathrm{y}}^{\mathrm{n}}+\Delta \omega_{z}^{\mathrm{n}}
\end{aligned}
$$

where $\Delta \omega_{\mathrm{x}}^{\mathrm{n}}, \Delta \omega_{\mathrm{y}}^{\mathrm{n}}, \Delta \omega_{\mathrm{z}}^{\mathrm{n}}$ are the angular velocity errors in navigation frame due to gyro measurement errors. The final velocity error equations can be written as:

$$
\begin{aligned}
& \delta \mathrm{f}_{\mathrm{y}}^{\mathrm{n}} \phi_{z}^{\mathrm{n}}-\mathrm{f}_{z}^{\mathrm{n}} \phi_{\mathrm{y}}^{\mathrm{n}}+\left(2 \Omega \mathrm{V}_{\mathrm{y}}^{\mathrm{n}} \cos \varphi+2 \Omega \mathrm{V}_{z}^{\mathrm{n}} \sin \varphi+\frac{\mathrm{V}_{\mathrm{x}}^{\mathrm{n}} \mathrm{V}_{\mathrm{y}}^{\mathrm{n}} \sec ^{2} \varphi}{\mathrm{R}_{\mathrm{N}}+\mathrm{h}}\right) \delta \varphi+ \\
& \frac{\mathrm{V}_{\mathrm{y}}^{\mathrm{n}} \tan \varphi-\mathrm{V}_{z}^{\mathrm{n}}}{\mathrm{R}_{\mathrm{N}}+\mathrm{h}} \delta \mathrm{V}_{\mathrm{x}}^{\mathrm{n}}+\left(2 \Omega \sin \varphi+\frac{\mathrm{V}_{\mathrm{x}}^{\mathrm{n}} \tan \varphi}{\mathrm{R}_{\mathrm{N}}+\mathrm{h}}\right) \delta \mathrm{V}_{\mathrm{y}}^{\mathrm{n}}-\left(2 \Omega \cos \varphi+\frac{\mathrm{V}_{\mathrm{x}}^{\mathrm{n}}}{\mathrm{R}_{\mathrm{N}}+\mathrm{h}}\right) \delta \mathrm{V}_{\mathrm{z}}^{\mathrm{n}}+\Delta \mathrm{f}_{\mathrm{x}}^{\mathrm{n}} \\
& \delta \mathrm{f}_{\mathrm{y}}^{\mathrm{n}} \phi_{\mathrm{x}}^{\mathrm{n}}-\mathrm{f}_{\mathrm{x}}^{\mathrm{n}} \phi_{z}^{\mathrm{n}}-\left(2 \Omega \mathrm{V}_{\mathrm{x}}^{\mathrm{n}} \cos \varphi+\frac{\left(\mathrm{V}_{\mathrm{x}}^{\mathrm{n}}\right)^{2} \sec ^{2} \varphi}{\mathrm{R}_{\mathrm{N}}+\mathrm{h}}\right) \delta \varphi-\left(2 \Omega \sin \varphi+\frac{2 \mathrm{~V}_{\mathrm{x}}^{\mathrm{n}} \tan \varphi}{\mathrm{R}_{\mathrm{N}}+\mathrm{h}}\right) \delta \mathrm{V}_{\mathrm{x}}^{\mathrm{n}}- \\
& \frac{\mathrm{V}_{z}^{\mathrm{n}}}{\mathrm{R}_{\mathrm{M}}+\mathrm{h}} \delta \mathrm{V}_{\mathrm{y}}^{\mathrm{n}}-\frac{\mathrm{V}_{z}^{\mathrm{n}}}{\mathrm{R}_{\mathrm{M}}+\mathrm{h}} \delta \mathrm{V}_{\mathrm{z}}^{\mathrm{n}}+\Delta \mathrm{f}_{\mathrm{y}}^{\mathrm{n}} \\
& { }^{(3)} \delta \mathrm{f}_{\mathrm{x}}^{\mathrm{n}} \phi_{\mathrm{y}}^{\mathrm{n}}-\mathrm{f}_{\mathrm{y}}^{\mathrm{n}} \phi_{\mathrm{x}}^{\mathrm{n}}-2 \Omega \mathrm{V}_{\mathrm{x}}^{\mathrm{n}} \sin \varphi \delta \varphi+\left(2 \Omega \cos \varphi+\frac{2 \mathrm{~V}_{\mathrm{x}}^{\mathrm{n}}}{\mathrm{R}_{\mathrm{N}}+\mathrm{h}}\right) \delta \mathrm{V}_{\mathrm{x}}^{\mathrm{n}}+\frac{2 \mathrm{~V}_{\mathrm{y}}^{\mathrm{n}}}{\mathrm{R}_{\mathrm{M}}+\mathrm{h}} \delta \mathrm{V}_{\mathrm{y}}^{\mathrm{n}}+\Delta \mathrm{f}_{\mathrm{z}}^{\mathrm{n}}
\end{aligned}
$$

where $\Delta \mathrm{f}_{\mathrm{x}}^{\mathrm{n}}, \Delta \mathrm{f}_{\mathrm{y}}^{\mathrm{n}}, \Delta \mathrm{f}_{\mathrm{z}}^{\mathrm{n}}$ are the errors in the specific acceleration due to accelerometers error in navigation frame. 


\subsection{Sensor error model}

In order to achieve the desired modeling accuracy, without unnecessarily increasing the complexity of the models and thereby the filters, it was determined that 6 unknown parameters for gyros and 6 unknown parameters for accelerometers are needed. The gyro drift rate reflects all the unknown gyro parameters such as (gyro scale factor, bias, misalignments, mass-unbalance, gravity square sensitivity, bias trends, gravity sensitivity trends, gyro torque nonlinearity). These parameters consists of those that are known to cause the gyros to drift as well as others such as axes misalignment that indirectly cause platform drift [8]. It is not necessary to model some of the other parameters but they must be compensated with values obtained from instrument testing [7]. All of the above unknown parameters undergo change as a function of time and therefore need to be estimated at the system level. Because of the complexity of the gyro error model so, we only focused on the two important factors which tends to gyro error measurements and we can consider all the above unknown parameters as a white noise $\left(\varepsilon_{g}\right)$. The two main factors are the gyro constant drift vector $\left(\boldsymbol{D}_{F}\right)$ and the gyro scale factor $\operatorname{vector}\left(S_{G}\right)$. Then the gyro measuring error can be modeled as:

$$
\begin{aligned}
& \Delta \omega_{\mathrm{x}}^{\mathrm{b}}=\mathrm{D}_{\mathrm{Fx}}+\mathrm{S}_{\mathrm{Gx}} \omega_{\mathrm{x}}^{\mathrm{b}}+\varepsilon_{\mathrm{gx}} \\
& \Delta \omega_{\mathrm{y}}^{\mathrm{b}}=\mathrm{D}_{\mathrm{Fy}}+\mathrm{S}_{\mathrm{Gy}} \omega_{\mathrm{y}}^{\mathrm{b}}+\varepsilon_{\mathrm{gy}} \\
& \Delta \omega_{\mathrm{z}}^{\mathrm{b}}=\mathrm{D}_{\mathrm{Fz}}+\mathrm{S}_{\mathrm{Gz}} \omega_{\mathrm{z}}^{\mathrm{b}}+\varepsilon_{\mathrm{gz}}
\end{aligned}
$$

where $\omega_{\mathrm{x}}^{\mathrm{b}}, \omega_{\mathrm{y}}^{\mathrm{b}}, \omega_{\mathrm{z}}^{\mathrm{b}}$ are the angular velocities in body frame measured by gyros, and $\varepsilon_{g}$ is the white noise vector. To calculate $\Delta \omega_{\mathrm{x}}^{\mathrm{p}}, \Delta \omega_{\mathrm{y}}^{\mathrm{p}}, \Delta \omega_{\mathrm{z}}^{\mathrm{p}}$ which represents the error in measuring angular velocities in platform coordinates system due to gyro errors and needed for attitude error equation. In north pointing mechanization $\left(\Delta \omega^{\mathrm{n}}=\Delta \omega^{\mathrm{p}}\right)$ then,

$$
\begin{aligned}
{\left[\begin{array}{c}
\Delta \omega_{\mathrm{x}}^{\mathrm{p}} \\
\Delta \omega_{\mathrm{y}}^{\mathrm{p}} \\
\Delta \omega_{\mathrm{z}}^{\mathrm{p}}
\end{array}\right] } & =\boldsymbol{C}_{\boldsymbol{b}}^{\boldsymbol{p}}\left[\begin{array}{l}
\mathrm{D}_{\mathrm{Fx}}+\mathrm{S}_{\mathrm{Gx}} \omega_{\mathrm{x}}^{\mathrm{b}}+\varepsilon_{\mathrm{gx}} \\
\mathrm{D}_{\mathrm{Fy}}+\mathrm{S}_{\mathrm{Gy}} \omega_{\mathrm{y}}^{\mathrm{b}}+\varepsilon_{\mathrm{gy}} \\
\mathrm{D}_{\mathrm{Fz}}+\mathrm{S}_{\mathrm{Gz}} \omega_{\mathrm{z}}^{\mathrm{b}}+\varepsilon_{\mathrm{gz}}
\end{array}\right] \\
& =\boldsymbol{C}_{\mathrm{b}}^{\mathrm{p}}\left[\begin{array}{l}
\mathrm{D}_{\mathrm{Fx}} \\
\mathrm{D}_{\mathrm{Fy}} \\
\mathrm{D}_{\mathrm{Fz}}
\end{array}\right]+\boldsymbol{C}_{\mathrm{b}}^{\mathrm{p}}\left[\begin{array}{ccc}
\omega_{\mathrm{x}}^{\mathrm{b}} & 0 & 0 \\
0 & \omega_{\mathrm{y}}^{\mathrm{b}} & 0 \\
0 & 0 & \omega_{\mathrm{z}}^{\mathrm{b}}
\end{array}\right]\left[\begin{array}{l}
\mathrm{S}_{\mathrm{Gx}} \\
\mathrm{S}_{\mathrm{Gy}} \\
\mathrm{S}_{\mathrm{Gz}}
\end{array}\right]+\boldsymbol{C}_{\mathrm{b}}^{\mathrm{p}}\left[\begin{array}{c}
\varepsilon_{\mathrm{gx}} \\
\varepsilon_{\mathrm{gy}} \\
\varepsilon_{\mathrm{gz}}
\end{array}\right]
\end{aligned}
$$

In the accelerometers error model we only focused on the two important factors which tends to 
accelerometer error measurements and we can consider the all entire reminder unknown parameters as a white noise vector

$(\bar{\nabla})$. The two main factors are the accelerometer bias vector $\left(\overline{\mathrm{K}}_{\mathrm{o}}\right)$ and the accelerometer scale factor vector $\left(\overline{\mathrm{k}}_{1}\right)$.Then the accelerometer measuring error can be modeled as [11]:

$$
\begin{aligned}
& \Delta \mathrm{f}_{\mathrm{x}}^{\mathrm{b}}=\mathrm{K}_{\mathrm{ox}}+\mathrm{K}_{1 \mathrm{x}} \mathrm{f}_{\mathrm{x}}^{\mathrm{b}}+\nabla_{\mathrm{x}} \\
& \Delta \mathrm{f}_{\mathrm{y}}^{\mathrm{b}}=\mathrm{K}_{\mathrm{oy}}+\mathrm{K}_{1 \mathrm{y}} \mathrm{f}_{\mathrm{y}}^{\mathrm{b}}+\nabla_{\mathrm{y}} \\
& \Delta \mathrm{f}_{\mathrm{z}}^{\mathrm{b}}=\mathrm{K}_{\mathrm{oz}}+\mathrm{K}_{1 \mathrm{z}} \mathrm{f}_{\mathrm{z}}^{\mathrm{b}}+\nabla_{\mathrm{z}}
\end{aligned}
$$

To calculate $\Delta \mathrm{f}_{\mathrm{x}}^{\mathrm{n}}, \Delta \mathrm{f}_{\mathrm{y}}^{\mathrm{n}}, \Delta \mathrm{f}_{\mathrm{z}}^{\mathrm{n}}$ which, represents the error in specific acceleration measurement due to accelerometer errors and needed for velocity error equations. Also, consider $\left(\Delta \mathrm{f}^{\mathrm{n}}=\Delta \mathrm{f}^{\mathrm{p}}\right)$ then,

$$
\begin{aligned}
{\left[\begin{array}{c}
\Delta \mathrm{f}_{\mathrm{x}}^{\mathrm{p}} \\
\Delta \mathrm{f}_{\mathrm{y}}^{\mathrm{p}} \\
\Delta \mathrm{f}_{\mathrm{z}}^{\mathrm{p}}
\end{array}\right]=} & \boldsymbol{C}_{\boldsymbol{b}}^{\boldsymbol{p}}\left[\begin{array}{l}
\mathrm{K}_{\mathrm{ox}}+\mathrm{K}_{1 \mathrm{x}} \mathrm{f}_{\mathrm{x}}^{\mathrm{b}}+\nabla_{\mathrm{x}} \\
\mathrm{K}_{\mathrm{oy}}+\mathrm{K}_{1 \mathrm{y}} \mathrm{f}_{\mathrm{y}}^{\mathrm{b}}+\nabla_{\mathrm{y}} \\
\mathrm{K}_{\mathrm{oz}}+\mathrm{K}_{1 \mathrm{z}} \mathrm{f}_{\mathrm{z}}^{\mathrm{b}}+\nabla_{\mathrm{z}}
\end{array}\right] \\
& =\boldsymbol{C}_{\boldsymbol{b}}^{\boldsymbol{p}}\left[\begin{array}{l}
\mathrm{K}_{\mathrm{ox}} \\
\mathrm{K}_{\mathrm{oy}} \\
\mathrm{K}_{\mathrm{oz}}
\end{array}\right]+\boldsymbol{C}_{\boldsymbol{b}}^{\boldsymbol{p}}\left[\begin{array}{ccc}
\mathrm{f}_{\mathrm{x}}^{\mathrm{b}} & 0 & 0 \\
0 & \mathrm{f}_{\mathrm{y}}^{\mathrm{b}} & 0 \\
0 & 0 & \mathrm{f}_{\mathrm{z}}^{\mathrm{b}}
\end{array}\right]\left[\begin{array}{l}
\mathrm{K}_{1 \mathrm{x}} \\
\mathrm{K}_{1 \mathrm{y}} \\
\mathrm{K}_{1 \mathrm{z}}
\end{array}\right]+\boldsymbol{C}_{\boldsymbol{b}}^{\boldsymbol{p}}\left[\begin{array}{c}
\nabla_{\mathrm{x}} \\
\nabla_{\mathrm{y}} \\
\nabla_{\mathrm{z}}
\end{array}\right]
\end{aligned}
$$

\subsection{GPS/GLONASS error model}

Since the global Navigation Satellite System (GLONASS) uses the PZ-90 frame but the GPS uses WGS-84 frame. So, in the integration of GPS/GLONASS it must be considered the interfacing and unification of the two systems [15]. In many literatures the GPS/GLONASS have a lot of error sources which affects on the accuracy of position and velocity. Even more, if most of these errors are corrected, still some random errors are existing. We can regard the random error as clock errors that are the first differential equation which describe the state errors can be written as:

$$
\mathbf{z}_{\mathrm{GPS}}=\mathbf{F}_{\mathrm{GPS}} \mathbf{x}_{\mathrm{GPS}}+\mathbf{w}_{\mathrm{GPS}}
$$




$$
\mathbf{x}_{\mathrm{GPS}}=\left[\begin{array}{llllll}
\delta \varphi_{\mathrm{G}} & \delta \lambda_{\mathrm{G}} & \delta \mathrm{h}_{\mathrm{G}} & \delta \mathrm{V}_{\mathrm{x}_{\mathrm{G}}} & \delta \mathrm{V}_{\mathrm{y}_{\mathrm{G}}} & \delta \mathrm{v}_{\mathrm{z}_{\mathrm{G}}}
\end{array}\right]^{\mathrm{T}}
$$

where $\delta \varphi_{\mathrm{G}}, \delta \lambda_{\mathrm{G}}, \delta \mathrm{h}_{\mathrm{G}}$ are the position errors of GPS and $\delta \mathrm{V}_{\mathrm{x}_{\mathrm{G}}}, \delta \mathrm{V}_{\mathrm{y}_{\mathrm{G}}}, \delta \mathrm{V}_{\mathrm{z}_{\mathrm{G}}}$ are the velocity errors of GPS.

We usually regard the position and velocity errors of GPS as first order Markov process noise, $\tau_{\varphi_{\text {GPS }}}, \tau_{\lambda_{\text {GPS }}}, \tau_{\mathrm{h}_{\text {GPS }}}, \tau_{\mathrm{Vx}_{\mathrm{CPP}}}, \tau_{\mathrm{V}_{\mathrm{Y}_{\mathrm{GPS}}}}, \tau_{\mathrm{V}_{\mathrm{Z} \text { GP }}}$ are Markov process time constants. From the above 3-models (INS error model, sensors error model, and GPS/GLONASS error model) we can construct the integration navigation system error model as follows[15]:

$$
\boldsymbol{x}(\mathrm{t})=\boldsymbol{F}(\boldsymbol{t}) \boldsymbol{x}(\mathrm{t})+\boldsymbol{G}(\mathrm{t}) \boldsymbol{w}(\mathrm{t})
$$

Where $\boldsymbol{x}(\mathrm{t}) \in \mathfrak{R}^{27}$ is the system state vector, $\boldsymbol{F}(\mathrm{t}) \in \mathfrak{R}^{27 \times 27}$ is the dynamic system matrix, $\boldsymbol{w}(\boldsymbol{t}) \in \mathfrak{R}^{12}$ is the system process noise vector, and $\boldsymbol{G}(\mathrm{t}) \in \mathfrak{R}^{27 \times 12}$ is the system noise matrix.

$$
\mathbf{F}(\mathrm{t})=\left[\begin{array}{ccc}
\mathbf{F}_{\text {INS }} & \mathbf{F}_{\text {sen. }} & 0_{9 \times 6} \\
0_{12 \times 9} & 0_{12 \times 12} & 0_{12 \times 6} \\
0_{6 \times 9} & 0_{6 \times 12} & \mathbf{F}_{\mathrm{GPS}}
\end{array}\right]
$$

Where $\mathbf{F}_{\mathrm{INS}}$ is the system dynamic matrix of navigation error model. The input system noise matrix can also be constructing as follows:

$$
\mathbf{G}(\mathrm{t})=\left[\begin{array}{ccc}
\mathbf{C}_{\mathrm{b}}^{\mathrm{p}} & 0_{3 \times 3} & 0_{3 \times 6} \\
0_{3 \times 3} & \mathbf{C}_{\mathrm{b}}^{\mathrm{p}} & 0_{3 \times 6} \\
0_{3 \times 3} & 0_{3 \times 3} & 0_{3 \times 6} \\
0_{12 \times 3} & 0_{12 \times 3} & 0_{12 \times 6} \\
0_{6 \times 3} & 0_{6 \times 3} & \mathbf{I}_{6 \times 6}
\end{array}\right]
$$

The measurement equation of the integrated INS/GPS/GLONASS navigation systems based on the position and velocity measurements. Then we can use the output from INS/GPS/GLONASS as the measurements. So, the measurement equation can be defined as follows [3]:

$$
\mathbf{z}=\mathbf{H x}+\mathbf{v}
$$

Where $\mathbf{H}$ is the measurement matrix and can be defined as:

$$
\mathbf{H}=\left[\begin{array}{cccccc}
0_{3 \times 3} & 0_{3 \times 3} & \mathbf{I}_{3 \times 3} & 0_{3 \times 3} & -\mathbf{I}_{3 \times 3} & 0_{3 \times 3} \\
0_{3 \times 3} & \mathbf{I}_{3 \times 3} & 0_{3 \times 3} & 0_{3 \times 3} & 0_{3 \times 3} & -\mathbf{I}_{3 \times 3}
\end{array}\right]
$$

and $\mathbf{x}$ is the observed measurement state and can be defined as: 


$$
\mathbf{X}=\left[\begin{array}{c}
\varphi_{\mathrm{INS}}-\varphi_{\mathbf{G}} \\
\lambda_{\mathrm{INS}}-\lambda_{\mathrm{G}} \\
\mathrm{h}_{\mathrm{INS}}-\mathbf{h}_{\mathrm{G}} \\
\mathbf{V}_{\mathbf{x}_{\mathrm{INS}}}-\mathbf{V}_{\mathbf{x}_{\mathbf{G}}} \\
\mathbf{V}_{\mathbf{y}_{\mathrm{INS}}}-\mathbf{V}_{\mathbf{y}_{\mathbf{G}}} \\
\mathbf{V}_{\mathbf{z}_{\mathrm{INS}}}-\mathbf{V}_{\mathbf{z}_{\mathbf{G}}}
\end{array}\right]=\left[\begin{array}{c}
\delta \varphi-\delta_{\varphi_{\mathbf{G}}} \\
\delta \lambda-\delta_{\lambda_{\mathbf{G}}} \\
\delta \mathbf{h}-\delta_{\mathbf{h}_{\mathbf{G}}} \\
\delta \mathbf{V}_{\mathbf{x}}-\delta_{\mathbf{V}_{\mathbf{G}}} \\
\delta \mathbf{V}_{\mathbf{y}}-\delta_{\mathbf{V}_{\mathbf{y}_{S}}} \\
\delta \mathbf{V}_{\mathbf{z}}-\delta_{\mathbf{V}_{\mathbf{z}_{\mathbf{G}}}}
\end{array}\right]
$$

and $v \in \Re^{6}$ is the measurement noise vector.

\subsection{Kalman filter functionality}

To use Kalman filter for the integration INS/GPS/GLONASS, we must discretize the continuous system matrix equation $[12,13]$. The discretization of the state equation and measurement equation are as follows:

$$
\begin{aligned}
& \boldsymbol{x}_{k}=\boldsymbol{\Phi}_{k, k-1} \boldsymbol{x}_{k-1}+\boldsymbol{\Gamma}_{k-1} \boldsymbol{w}_{k-1} \\
& z_{k}=H_{k}, \boldsymbol{x}_{k}+\boldsymbol{v}_{k}
\end{aligned}
$$

Where $\boldsymbol{\Phi}_{k, k-1}$ is the system discrete matrix, $\boldsymbol{\Gamma}_{k-1}$ is the discrete system noise process.

$$
\begin{aligned}
& \boldsymbol{\Phi}_{k, k-1}=\sum_{n=0}^{\infty} \frac{\left[\mathbf{F}\left(\mathrm{t}_{k}\right) \mathrm{T}\right]^{n}}{\mathrm{n} !} \\
& \boldsymbol{\Gamma}_{k-1}=\left\{\sum_{n=1}^{\infty}\left[\frac{1}{\mathrm{n} !}\left(\boldsymbol{F}\left(\mathrm{t}_{k}\right) \mathrm{T}\right)^{n-1}\right]\right\} \boldsymbol{G}\left(\mathrm{t}_{k}\right) \mathrm{T}
\end{aligned}
$$

where $\mathrm{T}$ is the interval time, in the real time we use some definite time

- Time update

$$
\begin{aligned}
& \hat{\boldsymbol{x}}_{k / k-1}=\boldsymbol{\Phi}_{k, k-1} \hat{\boldsymbol{x}}_{k-1} \\
& \mathbf{P}_{k / k-1}=\boldsymbol{\Phi}_{k, k-1} \mathbf{P}_{k-1} \boldsymbol{\Phi}_{k, k-1}^{T}+\boldsymbol{\Gamma}_{k-1} \boldsymbol{Q}_{k-1} \boldsymbol{\Gamma}_{k-1}^{T}
\end{aligned}
$$

- Measurement update

$$
\begin{aligned}
& \mathbf{K}_{k}=\mathbf{P}_{k / k-1} \boldsymbol{H}_{k}^{T}\left(\boldsymbol{H}_{k} \mathbf{P}_{k / k-1} \boldsymbol{H}_{k}^{T}+\boldsymbol{R}_{k}\right)^{-1} \\
& \hat{\boldsymbol{x}}_{k}=\hat{\boldsymbol{x}}_{k / k-1}+\mathbf{K}_{k}\left(z_{k}-\boldsymbol{H}_{k} \hat{\boldsymbol{x}}_{k / k-1}\right) \\
& \mathbf{P}_{k}=\left(\boldsymbol{I}-\mathbf{K}_{k} \boldsymbol{H}_{k}\right) \mathbf{P}_{k / k-1}
\end{aligned}
$$

\section{Simulation Results}

Sampling time of trajectory data is chosen as $10 \mathrm{~ms}$, INS computation interval time $20 \mathrm{~ms}$, 
GPS/GLONASS transfer data frequency $1 \mathrm{HZ}$, and Kalman filter interval time $1 \mathrm{sec}$. The initial attitude error angle is chosen as 500", initial velocity error $1.0 \mathrm{~m} / \mathrm{s}$, initial error of latitude and longitude angle $0.15 \mathrm{rad}$, initial altitude $1000 \mathrm{~m}$, initial accelerometer bias error $10^{-8} \mathrm{~m} / \mathrm{s}^{2}$, initial accelerometer scale factor error $10^{-8}$, initial gyro fixed drift error $10^{-8} " / \mathrm{s}^{2}$, initial gyro scale factor error $10^{-8}$, random drift of each gyro $0.1^{\circ} / \mathrm{h}$, and random bias of each accelerometer $10 \mu \mathrm{g}$. Markov time constant $\tau=60 \mathrm{sec}$. Fig(3) shows INS estimated error parameters.

Markov time constant $\tau=60 \mathrm{sec}$. Fig(3) shows INS estimated error parameters.
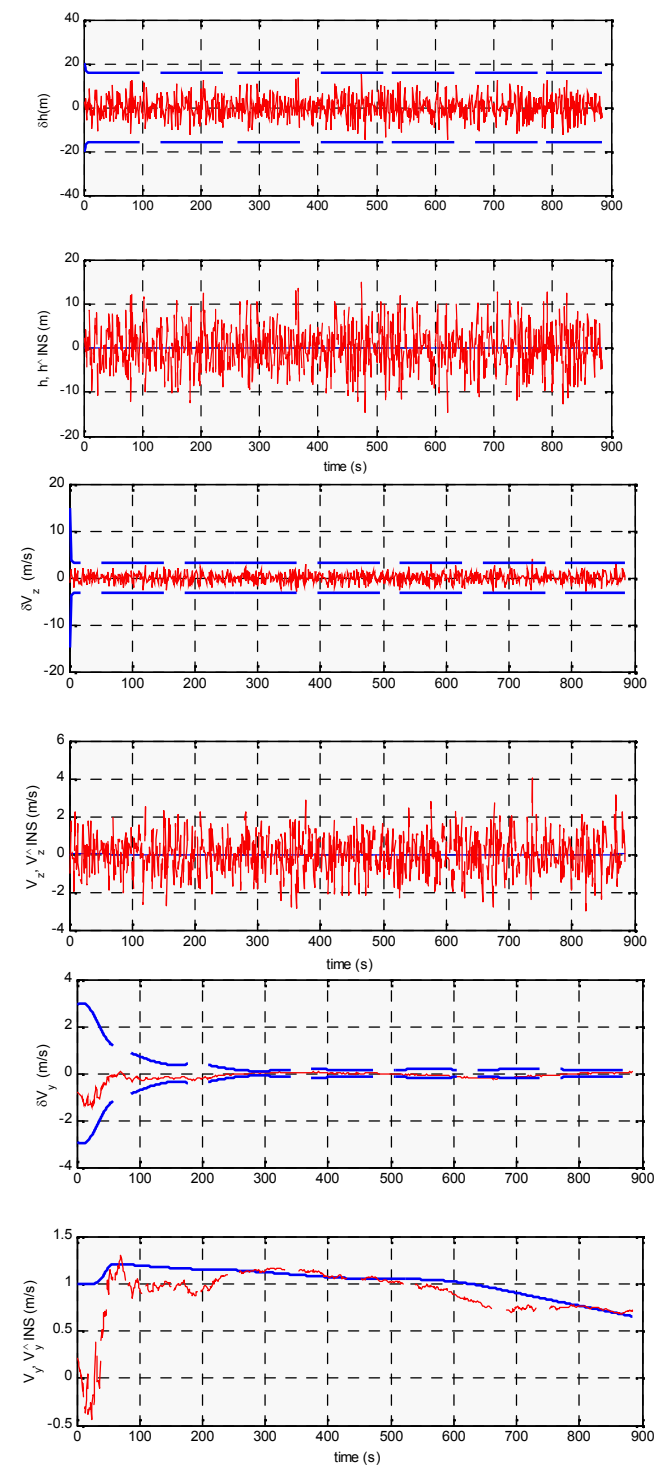

Figure (3): Estimated Errors of INS Parameters
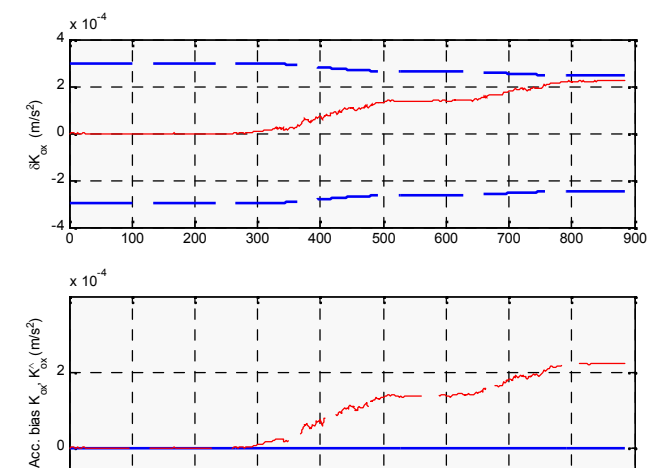

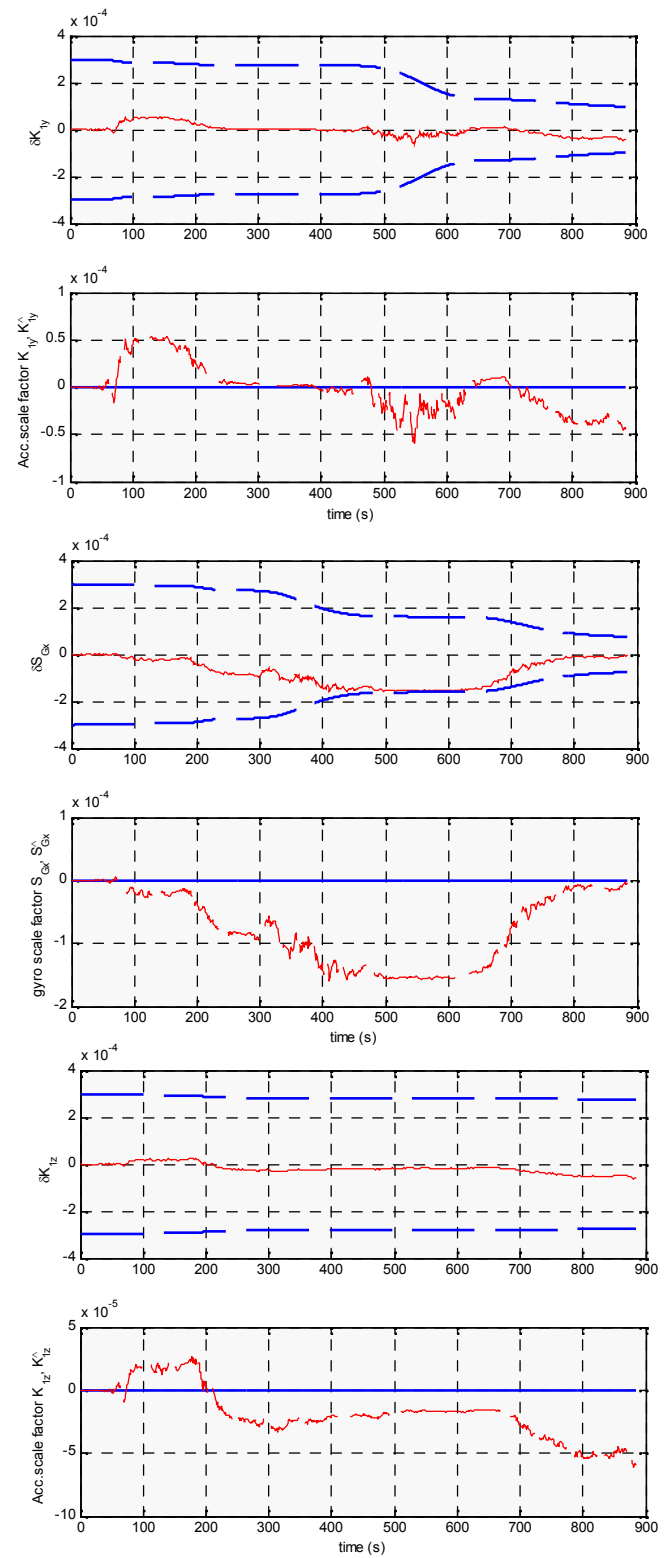

Figure (4): Estimated Errors of Sensors Parameters
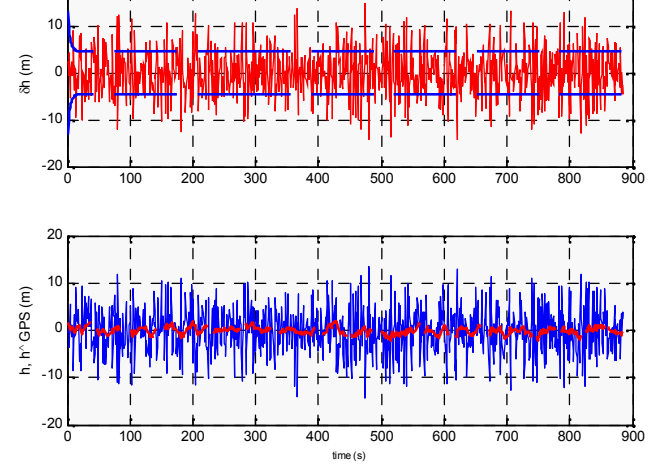

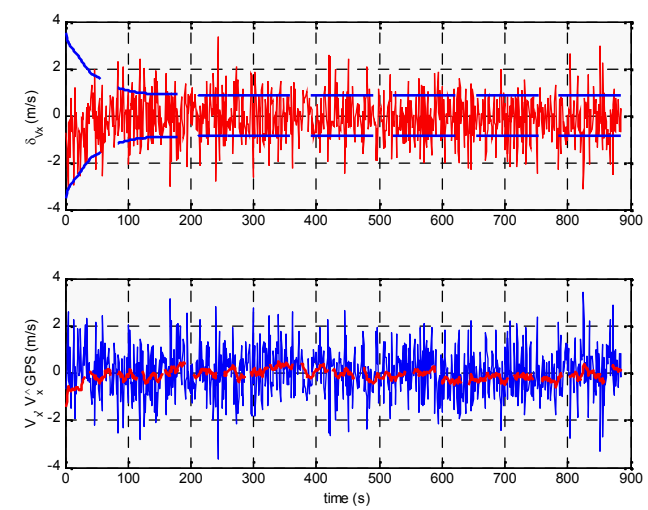

Figure (5): GPS Estimated Errors

\section{Conclusions:}

In this paper we demonstrated the importance of the INS/GPS/GLONASS integration into ballistic navigation solution. The SINS uses (ENU) frame and the integrated system uses position and velocity as measurements. The system model of the integrated system for Kalman filtering are derived and modeled as 27-states. This state estimation system shown clearly the application of fundamental modeling and filtering techniques. The simulation is built on the integrated system INS/GPS/GLONASS and the trajectory generator data. From the results we found that some parameters of estimated gyro errors such as vertical and east gyro drifts, and also estimated east accelerometer bias are not observables.

\section{References:}

[1] George M. Siouris. “Aerospace Avionics Systems” A modern Synthesis 1993.

[2] M.E. Canon, R. Nayak, G. Lachapelle "Low Cost INS/GPS Integration Concepts and Testing", Institute of navigation National Technical Meeting/ Anaheim, CA/ January 26-28,2000.

[3] Saurabh Godha, "Strategies for GPS/INS Integration", Department of Geometrics Engineering Calgary University, ENGO $62327^{\text {th }}$ Feb. 2004.

[4] Rodeny E. Stubbs, Whittak H. Huang and Eric Schimtz, "Integration of GPS into Ballistic Navigation Solution", Lockheed Martin Astronautics By American Institute of Aeronaumatic and Astronautics, Inc. 1997. 
[5] Ibrahim I. Arafa, M. I. Latif, Zhong .H.Yue "Correction of the Flight Path Based on Error Estimation Of SINS Ground Alignment Using Modern Computer Techniques", Beihang University, Beijing 100083, Conferences second Asian workshop of foundations of software. Nanjing China DEC. 2003

[6] Hao Shuguang, "Study on SINS Initial Alignment and Integration Navigation Techniques", Master degree, Beijing University of Aeronautics and astronautics March 2004.

[7] M.S. Grewal, V.D. Henderson "Application of Kalman Filtering to the Calibration and Alignment of Inertial Navigation Systems", $29^{\text {th }}$ conference on decision and control IEEE 1990.

[8] O.S. Salychev, V. V. Voronov "Low Cost INS/GPS Integration Concept and Testing" Institute of navigation National Technical Meeting/ Anaheim, CA/January 26-28 2000.

[9] Kline, Paul, Vangraas, Frank (Ohio Univ., Athens, OH, United States) "Integrated Inertial/ GPS" NASA Center for Aerospace Information (CASI), 1990.

[10] Wolf, R., G.W. Hein, B. Eissfeller and Loeehnert, "An Integrated Low Cost GPS/INS Attitude Determination and Position Location System", Proceedings of GPS-1996, Meeting of the Institute of navigation, Ed., Kansas City, Missouri, 1996, pp.975-981.

[11] Wang. J. (1998a). "Mathematical models for combined GPS and GLONASS positioning" $11^{\text {th }}$ Int. Tech. Meeting of Satellite Division of the U.S. Inst. Of Navigation GPS IOV'98, Nashville, Tennessee, 15-18 September, 1333-1344.

[12] James A. Slater, National Imagery and Mapping Agency, USA Pascal Willis, University FAF Munich, Germany "The International GLONASS Experiment (IGEX-98): Organization, Preliminary Results and Future Plans", 1999.

[13] Wei Chun ling, PhD thesis "Alignment of Inertial navigation and Terrain systems", Beijing University of Aeronautics and Astronaumatic, Beijing, China, 100083, 2001.

[14] B. Azimi-Sadjadi, and P. S. Krishnaprasad Rensselaer Polytechnic Institute Troy, New York, 12180 "Approximate Nonlinear Filtering and its Application in Navigation", University of Maryland at College Park, MD 20742, March, 2002.

[15] Yee, Robert (NASA Ames Research Center, Moffett Field, CA United States) "Inertial Pointing and Positioning System" NASA Center for Aerospace Information (CASI), United States 1998.

[16] Kevin. J. Walchko "Inertial Navigation" University of Florida, Gainesville, FL 32611-6200, NASA Goddard Space Flight Center, 2002 Florida Conference on Recent Advances in Robotics. 